pédagogie

\title{
Formation à la recherche et développement professionnel des paramédicaux
}

JEAN-PHILIPPE

DILLENSEGER $^{\mathrm{a}}$

Merm de formation, professeur certifié au lycée Jean-Rostand de Strasbourg - section IMRT (formation initiale des Merm),

docteur en physique-

biophysique, membre du laboratoire ICube (équipes MMB/AVR) - UMR 7357 CNRS

Marion Coquand-

GANDIT $^{\mathrm{b}}$

Merm de formation, cadre formatrice à I'Ifmem de Grenoble (formation initiale des Merm), docteur en physiopathologiepharmacologie, chercheuse associée au LaRAC

(Laboratoire de recherche

sur les apprentissages en contexte-EA602)

Yann Le FaOU ${ }^{\text {c,* }}$

Merm de formation, coordonnateur de la recherche à I'IFPEK Rennes, doctorant en sciences de l'éducation au Centre de recherche sur les enseignements, les apprentissages et la didactique (CREAD) EA 3875

aLycée Jean-Rostand, 5, rue Edmond-Labbé, 67084 Strasbourg cedex, France

$\mathrm{b}_{\text {Ifmem, CHU Grenoble Alpes, }}$

19, avenue de Kimberley, CS 90338, 38434 Échirolles Cedex, France

Ifpek, 12, rue Jean-LouisBertrand, 35000 Rennes, France

\footnotetext{
* Auteur correspondant. Adresse e-mail : y.lefaou@ifpek.org (Y. Le Faou).
}

\begin{abstract}
- Avec la réforme de la formation initiale des professions paramédicales, la formation à et par la recherche est présentée comme un facteur d'évolution de la pratique professionnelle Dans le métier de manipulateur d'électroradiologie médicale, il est essentiel d'intégrer des résultats de la recherche scientifique dans la pratique clinique afin d'améliorer la qualité de la prise en charge des patients.

๑ 2019 Publié par Elsevier Masson SAS

Mots clés - développement professionnel ; evidence-based practice ; formation ; manipulateur d'électroradiologie médicale ; recherche paramédicale
\end{abstract}

Training in research and professional development of paramedic. With reforms of paramedical professions curricula, training in and through research is presented as a factor that impact professional practice. For radiographers, it is essential to integrate the results of scientific research in their clinical practice to increase patient care quality.

(c) 2019 Published by Elsevier Masson SAS

Keywords - evidence-based practice; paramedical research; professional development; radiographers; training

a recherche est un univers Eencore nouveau, méconnu, présenté parfois comme non compatible avec les représentations anciennes, mais encore tenaces, de la profession de manipulateurs d'électroradiologie médicale (Merm). Le développement d'une "recherche paramédicale" et la "formation par la recherche" sont des concepts nouveaux [1], encore mal compris du terrain. L'évolution vers la recherche est pourtant incontournable pour l'évolution des professions paramédicales qui se doivent actuellement de rattraper le retard de la France dans ce domaine par rapport à d'autres pays. L'évolution de la profession ne pourra se faire sans le développement d'une recherche structurée, acceptée, et concrétisée par des écrits et des coopérations au niveau national et international [2].
Pour se diriger sereinement vers ces objectifs, il nous a semblé utile d'éclairer les professionnels sur la situation actuelle.

\section{ÉTAT DES LIEUX DES CHERCHEURS PARAMÉDICAUX}

I Dans le monde anglo-saxon, des fonctions d'enseignement et de recherche au niveau universitaire sont occupées depuis le début des années 1980 par des praticiens paramédicaux en charge de développer des axes de recherche spécifiques aux professions de santé. Les sciences infirmières et les métiers de la rééducation sont des professions pionnières dans ce domaine au niveau international. Depuis plus d'une dizaine d'années, des Merm (radiotechnologist, radiographers), principalement dans le monde anglo-saxon, suivent cette voie et occupent progressivement des postes d'enseignants-chercheurs à l'université.

Le doctorat est le prérequis indispensable à l'accès à ces fonctions ; la part de Merm titulaires de doctorats est en forte progression en Europe. L'European Federation of Radiographer Societies (EFRS) a ainsi comptabilisé 14 universités qui proposent des parcours de doctorat $(\mathrm{PhD})$ spécifiques aux Merm [3,4].

I En France, il n'existe actuellement pas d'école doctorale (ED) spécifique aux sciences paramédicales. Cependant, certains paramédicaux titulaires de master et ayant une expérience en termes de publication scientifique ont pu intégrer des programmes de troisième cycle. L'objectif pour ces professionnels est de trouver un sujet de thèse, qui permettra de développer des savoirs scientifiques et des compétences 
TABLAU 1. État des lieux du nombre et de la fonction de paramédicaux-docteurs en France (chiffres indicatifs donnés par les associations professionnelles à la date du 30 mai 2018).

\begin{tabular}{|c|c|c|c|c|}
\hline $\begin{array}{l}\text { Formation initiale (nombre } \\
\text { de professionnels recensés) }\end{array}$ & $\begin{array}{l}\text { Nombre de docteurs et } \\
\text { doctorants estimés en } 2017 \\
\text { (nombre de docteurs pour } \\
1000 \text { professionnels) }\end{array}$ & $\begin{array}{l}\text { Nombre de disciplines } \\
\text { académiques }\end{array}$ & $\begin{array}{l}\text { Titulaires } \\
\text { d'une habilitation } \\
\text { à diriger des } \\
\text { recherches (HDR) }\end{array}$ & Postes universitaires \\
\hline $\begin{array}{l}\text { Masseurs-kinésithérapeutes } \\
(86500)\end{array}$ & $\begin{array}{l}\approx 150 \\
(1,7 / 1000)\end{array}$ & 10 & 10 & $\begin{array}{l}10 \mathrm{MCF} \\
4 \mathrm{PR}\end{array}$ \\
\hline $\begin{array}{l}\text { Infirmiers } \\
(640000)\end{array}$ & $\begin{array}{l}\approx 150 \\
(0,2 / 1000)\end{array}$ & 10 & 3 & $3 \mathrm{MCF}$ \\
\hline $\begin{array}{l}\text { Orthophonistes } \\
(24000)\end{array}$ & $\begin{array}{l}\approx 15 \\
(0,6 / 1000)\end{array}$ & 3 & 1 & $\begin{array}{l}5 \mathrm{MCF} \\
1 \mathrm{PR}\end{array}$ \\
\hline $\begin{array}{l}\text { Ergothérapeutes } \\
(11200)\end{array}$ & $\begin{array}{l}\approx 15 \\
(1,4 / 1000)\end{array}$ & 5 & / & pas d'universitaires connus \\
\hline $\begin{array}{l}\text { Psychomotriciens } \\
(11000)\end{array}$ & $\begin{array}{l}\approx 10 \\
(0,9 / 1000)\end{array}$ & non renseigné & / & $2 \mathrm{MCF}$ \\
\hline $\begin{array}{l}\text { Pédicures- } \\
\text { Podologues } \\
\text { (12000) }\end{array}$ & $\begin{array}{l}\approx 10 \\
(0,9 / 1000)\end{array}$ & non renseigné & 0 & 0 \\
\hline $\begin{array}{l}\text { Merm } \\
(35000)\end{array}$ & $\begin{array}{l}n<10 \\
(<0,3 / 1000)\end{array}$ & non renseigné & 0 & 0 \\
\hline
\end{tabular}

MCF : maître de conférences; Merm : manipulateur d'électroradiologie médicale; PR : professeur des universités.

méthodologiques transférables aux champs disciplinaires de sa profession.

Le tableau 1 indique le nombre approximatif de docteurs / doctorants paramédicaux en France dans chaque profession. Obtenir des chiffres précis est difficile puisqu'il n'existe pas de recensement officiel. Ces chiffres indicatifs révèlent cependant le retard de la profession de Merm à la fois par rapport aux autres professions paramédicales en France, mais égalementà la situation des Merm dans le monde. À titre d'exemple, au RoyaumeUni, le taux de un docteur pour 100 manipulateurs devrait vraisemblablement être atteint dans les prochaines années; plus de 150 docteurs sont actuellement dénombrés chez nos collègues britanniques [5]. Par ailleurs, parmi les rares Merm français titulaires d'une thèse, on observe qu'une proportion importante "quitte" la profession pour occuper des places d'ingénieurs ou rejoindre le milieu de la physique médicale en suivant la formation menant au diplôme de qualification en physique radiologique et médicale (DQPRM). Il s'agit alors davantage d'une voie de réorientation qu'une évolution dans la profession. Cette "fuite" est regrettable pour l'émancipation de la profession ; elle s'explique en partie par la difficulté de valoriser la détention d'un doctorat chezles paramédicaux en France.

\section{INTÉRÊT \\ DE L'UNIVERSITARISATION}

I Même si l'universitarisation de la formation initiale des Merm est engagée, elle ne correspond actuellement qu'à des conventionnements à l'échelle locale. D'après le sociologue Raymond Bourdoncle [6], il existe une universitarisation complète lorsque «les instituts de formation d'origine disparaissent au profit des structures universitaires habituelles et de leurs modes ordinaires de fonctionnement; les savoirs professionnels y sont non seulement transmis, mais aussi créés et accumulés avec une large place à l'activité de recherche; les personnels de formation s'orientent vers un nouveau statut, celui d'enseignant-chercheur, qui exige un doctorat poury accéder ».

Iinsi, si l'on suit cette logique, l'étape suivante de l'universitarisation demanderait la création de départements ou d'instituts universitaires pilotant sur un plan scientifique et pédagogique les structures de formation actuelles (instituts de formation de Merm [Ifmem], lycées). Ce passage impliquerait, tout d'abord, un partage de gouvernance entre l'université et les établissements de formation ; puis la création d'un corps d'enseignants-chercheurs, qualifiés par le conseil national des universités (CNU), issus de ces professions (Mermdocteurs). Même si des pistes semblent ouvertes, elles restent encore au stade de la réflexion et de la négociation interstructurelles. L'ouvrage La formation entre universitarisation et professionnalisation. Tensions et perspectives dans des métiers de l'interaction humaine, paru en 2018, illustre bien le contexte actuel [7]. Cependant, en cette

\section{RÉFÉRENCES}

[1] Debout C, Eymard C Rothan-Tondeur M. Une formation doctorale dans la filière infirmière : plus-value et orientations dans le contexte français. Rech Soins Infirm. 2010;(100):134-44. [2] Dillenseger JP. Être manipulateur d'électroradiologie médicale à l'horizon 2025. Soins Cadres. 2016;25(97):43-6. [3] McNulty JP, Rainford L, Bezzina $P$ et al. A picture of radiography education across Europe. Radiography. 2016;22(1):5-11. [4] England A, Geers-van Gemeren S et al. Clinical radiography education across Europe. Radiography. 2017;23 Suppl 1:S7-15.

[5] Snaith B, Harris MA, Harris R. Radiographers as doctors: A profile of UK doctoral achievement. Radiography. 2016;22(4):282-6

[6] Bourdoncle R. "Universitarisation". Recherche et formation. 2007;(54):135-49. [7] Adé D, Piot T. La formation entre universitarisation et professionnalisation. Tensions et perspectives dans des métiers de l'interaction humaine. Rouen: PURH; 2018. 
TABleau 2. Critères de différenciation entre profession et semi-profession (d'après [9]).

Profession
1. Les professions rendent à la personne et à la société
des services essentiels.
2. Chaque profession est attachée à un domaine
de besoins bien identifié.
3. Une profession possède un corpus propre
de connaissances et de savoir-faire.
4. Les décisions professionnelles se prennent en accord
avec des connaissances, des principes, des théories
validées et confirmées.
5. La profession repose sur des disciplines
fondamentales dont elle tire son corpus
de connaissances et de savoir-faire.
6. Les associations professionnelles contrôlent les
conditions du travail des professionnels (par exemple
I'admission dans la profession, les normes
professionnelles, l'autorisation d'exercer).
7. Il existe des normes pour être admis dans
la profession et pour continuer de l'exercer.
8. La préparation et l'intégration à la profession
supposent une formation de longue durée, généralement
à l'université ou dans une école professionnelle
universitaire.

1. Un métier de statut inférieur.

2. Des périodes de formation plus courtes.

3. Une absence de reconnaissance par la société de ce que la nature du service rendu ou le niveau de compétence atteint justifie l'autonomie accordée usuellement aux professions.

4. Un corpus de connaissances et de savoir-faire moins spécialisé et moins hautement développé.

5. Une tendance chez l'individu à s'identifier à l'institution qui l'emploie plutôt qu'à la profession elle-même.

6. Une exposition plus grande à la surveillance et au contrôle de l'administration et des instances de tutelle. 7. Une moindre autonomie dans la prise de décision professionnelle et une responsabilité devant les supérieurs plutôt que devant la profession. 8. Une gestion par des personnes qui ont été ellesmêmes formées à cette semi-profession et l'ont pratiquée.

9. L'absence d'un droit de communication privilégiée entre le professionnel et l'usager.

Certaines filières paramédicales considérées au niveau sociologique comme des semi-professions acquièrent progressivement des critères de "professions".

\section{RÉFÉRENCES}

[8] Etzioni WA. The Semi-

Professions and Their

Organization: Teachers, Nurses, Social Workers. New York (ÉtatsUnis): Free Press; 1969.

[9] Chevallard Y. L'échec splendide des IUFM et l'interminable passion du pédant. Quel avenir pour le métier de professeur ? Regards des didactiques des disciplines sur les pratiques et la formation des enseignants. Toulouse; 2010. [10] Everaere C. Proposition d'un outil d'évaluation de l'autonomie dans le travail. Rev Fr Gest. 2007;(180):45-59. [11] Foray P. Autonomie. Le Télémaque. 2017;(51):19-28. [12] Freidson E. La profession médicale. Paris: Payot; 1984. [13] Novic M. Développement de compétences par la recherche. Rech Soins Infirm. 2010;(102):83-93.

[14] Rinck F. Former à (et par) l'écrit de recherche. Quels enjeux, quelles exigences ? Le français aujourd'hui. $1^{\text {er }}$ septembre 2011;(174):79-89. période charnière d'“arbitrages", la cible qu'est l'universitarisation desformations paramédicalesfait aujourd'hui consensus et permettra de voir l'émergence progressive de chercheurs paramédicaux dans les différentes filières venant ainsi rattraper progressivement le retard français dans ce domaine.

I Ce vivier facilitera et impulsera théoriquement la diffusion de travaux au niveau national et international et se rapprochera des standards "universitaires" (partage et diffusion des savoirs). En effet, rares sont les travaux publiés par des Merm dans des revues professionnelles référencées (Radiography, Journal of Nuclear Medicine Technology, Journal of Medical Imaging and Radiation Sciences, etc.). Cette situation restreint le rayonnement des travaux français à l'échelle internationale ainsi que la visibilité sur la manière dont s'organise et évolue la profession. Les pays les plus impliqués et productifs en matière de publications et de coopérations internationales sont ceux qui disposent d'un nombre significatif de Mermdocteurs impulsant une dynamique dans les établissements hospitaliers et/ou de formation. Cette recherche, développée par et pour les Merm, accompagnera ainsi naturellement l'évolution de la profession vers davantage d'autonomie et de réflexivité.

\section{LA RECHERCHE \\ COMIME FACTEUR \\ D'Évolution}

I La recherche et le travail d'écriture sont souvent présentés comme des moteurs de la réflexivité. Dès le premier article du référentiel de formation initiale des paramédicaux, il est indiqué que la finalité est de former "un professionnel autonome, responsable et réflexif”. L'outil "recherche" permet donc de transformer le métier en profession instituée, socialement reconnue. Sur le plan sociologique, les paramédicaux (les professions du "travail sur autrui" en général) ne sont pas considérées comme des professions, mais comme des semi-professions [8] dont les critères sont présentés dans le tableau 2 [9]. Néanmoins les ambitions actuelles des représentants des professions "paramédicales", portées par la démarche d'universitarisation, tendent à vouloir modifier cette représentation.

I La mutation d'une semiprofession vers une profession s'effectue souvent par l'acquisition d'une autonomie professionnelle réelle, définie comme "pouvoir d'agir en situation" [10]. Cette autonomie peut être décrite à différents niveaux [11] dans lesquels la réflexion (définie souvent comme "pensée critique”) a une place prépondérante dans le processus d'autonomisation. Dans les professions de santé, la recherche est décrite comme étant le moyen par excellence d'acquérir cette pensée critique [12,13].

\section{L'initiation à la recherche} intègre ainsi les actuels et récents référentiels de formation (paramédicaux, mais aussi dans le secteur médicosocial) pour accompagner cette volonté, ambitieuse mais légitime, de transformation sociologique de métiers qui s'engagent fortement dans cette démarche. Les apports de la formation à et par la recherche sont multiples : questionnements, méthode, rigueur, gestion de projet, formation à l'écriture, à la publication, à la valorisation, à la pensée autonome, à l'analyse critique de l'information [14,15]. Une étude américaine prospective montre, d'ailleurs, que pour obtenir une progression significative du niveau de santé de la population et pour que l'impact de la profession sur ce niveau de santé soit mesurable, il faudrait atteindre un niveau 
de $2 \%$ de docteurs au sein d'un corps professionnel [16]. Dans nos sociétés où le poids du diplôme, et la valeur sociale qui lui est attribuée, sont prépondérants, créer un contingent de paramédicaux-docteurs serait un facteur d'attractivité complémentaire du métier [17].

\section{UN MOYEN INTÉGRATEUR DES RÉSULTATS ACTUELS DE LA SCIENCE}

I L'évolution d'un exercice professionnel ne s'effectue pas sans une progression des connaissances contributives au domaine de pratique associé. La méthode scientifique est le modèle dominant de la construction de la connaissance depuis le XvII ${ }^{\mathrm{e}}$ siècle avec la "découverte" de la "médecine expérimentale" décrite précisément par Claude Bernard en 1865 dans son célèbre traité [18]. Une profession doit donc maîtriser son domaine scientifique (la discipline académique) pour fabriquer un nouveau savoir, applicable au champ de pratique (le métier) ; on parle alors de pratique fondée sur des faits / preuves ou d'Evidence-Based Practice (EBP) [19] fortement développées en médecine [20] et plus récemment chez les paramédicaux [21].

Chez les Merm aussi, des articles récents traitant de thématiques professionnelles (radioprotection, hygiène, etc.) témoignent de la nécessité de développer et d'intégrer une EBP caractéristique de la profession [22,23]. Il s'agit avec cette approche de former des professionnels, des praticiens réflexifs [24], plus que de développer des chercheurs au sens académique. En effet, l'EBP est avant tout une stratégie de formation à l'esprit critique, mais favorise en même temps, pour ces promoteurs, l'intégration des données de la science dans les pratiques professionnelles quotidiennes.

\section{UN MOYEN D'ÉMANCIPATION DE LA PROFESSION}

Les compétences acquises par la recherche apportent une capacité d'argumenter dans son propre champ professionnel. En effet, cette autonomie partagée est nécessaire en connaissance des éléments de justification de la pratique dite "scientifique" (EBP). Le professionnel utilise alors les mêmes outils conceptuels, méthodologiques et scientifiques que les "professions". Cependant le terme d'émancipation ne doit pas être assimilé à un éloignement vis-à-vis du corps médical. Au contraire, cette émancipation estavant tout intraprofessionnelle et culturelle; elle stimulerait la réflexivité, ce qui apporterait une amélioration qualitative et quantitative des connaissances professionnelles propres. Elle viendrait naturellement renforcer la qualité des coopérations avec le corps médical et les autres corps de métiers. En même temps, cela enrichirait la pratique dans un souci commun d'innovation et d'amélioration, de la qualité et de la sécurité dans la prise en charge des patients.

\section{Conclusion}

Trois axes de développement de la profession par la recherches sont essentiels :

- les chercheurs dans une profession peuvent être vus comme des "marginaux sécants" [25], comme des innovateurs socioprofessionnels [26] ;

- pour favoriser une formation à la recherche (grade licence/mas- ter) à l'échelle d'un groupe professionnel, il est incontournable de disposer de Merm, de cadres et de formateurs, titulaires d'un doctorat (formation par et pour la recherche);

- le potentiel d'émancipation que possède la recherche, par, entre autres, la participation des professionnels eux-mêmes à l'évolution des connaissances dans leur propre champ d'activité (imagerie médicale, imagerie interventionnelle, radiothérapie, etc.).

Tous ces mécanismes d'évolution de la profession demandent un engagement politique fort et conjoint, impliquant les représentants institués de la profession (associations) et le milieu étudiant (futurs professionnels), le tout appuyé par les différentes gouvernances en charge de la formation initiale et avancée. $\mathrm{Si}$, sur ce dernier point, le projet ministériel vise à promouvoir l'émergence de la recherche paramédicale, cette entreprise nécessite également et fondamentalement l'adhésion des professionnels de terrain.

La recherche chez les paramédicaux n'est qu'une des voies possibles proposée dans le rapport sur la Stratégie nationale de transformation du système de santé 2018-2022 [27], mais qui incombe finalement directementàl'acteur, le professionnel, maîtrisant ainsi son propre avenir. L'engagement dans des troisièmes cycles semble aujourd'hui fondamental pour faire évoluer les pratiques. Il importe à chaque professionnel de comprendre et d'adhérer à cette démarche collective pour favoriser l'émergence progressive (car cela prendra du temps), mais nécessaire, de futurs chercheurs qui accompagneront l'évolution de la profession vers plus d'attractivité tout en poursuivant l'amélioration de la qualité de prise en charge des patients.

\section{RÉFÉRENCES}

[15] Mias C, Piaser A.

La formation "à" et "par"

la recherche : une voie

de professionnalisation? Examen

de représentations d'étudiants

en master "Recherche".

Les dossiers des sciences de l'éducation. 2015;(34):53-74.

[16] Aiken LH. Nurses

for the future. N Engl J Med.

2011;364(3):196-8.

[17] DubetF, Duru-Bellat M,

VérétoutA. Les sociétés

et leur école. Emprise du diplôme et cohésion sociale. Paris: Points; 2015.

[18] Bernard C. Introduction à l'étude de la médecine expérimentale. Paris: Éditions Garnier-Flammarion; 1966. [19] McKibbon KA. Evidencebased practice. Bull Med Libr Assoc. 1998;86(3):396-401. [20] Sackett DL, Rosenberg WMC, Gray JAM et al. Evidence based medicine: what it is and what it isn't. BMJ. 1996;312(7023):71-2. [21] Poiroux L. L'intégration des données probantes en pratique clinique. Réalités et enjeux d'une démarche d'Evidence based nursing. Paris: De BoeckEstem; 2015.

[22] Upton D, Upton P. Knowledge and use of evidence-based practice by allied health and health science professionals in the United Kingdom. J Allied Health. 2006;35(3):127-33.

[23] Harris R, Paterson A. Exploring the research domain of consultant practice: perceptions and opinions of consultant radiographers. Radiography. 2016;22(1):12-20. [24] Schön DA. Le praticien réflexif. À la recherche du savoir caché dans l'agir professionnel. Montréal (Canada): Éditions Logiques; 1994. [25] Becker HS. Outsiders. Études de sociologie de la déviance. Paris: Éditions Métailé; 1963.

[26] Le Faou Y. Recherche paramédicale et reconnaissance au travail dans les établissements de santé. Soins Cadres.

2017;26(102):47-9.

[27] Ministère des Solidarités et de la Santé. Stratégie nationale de santé 2018-2022. 2017. https:// solidarites-sante.gouv.fr/IMG/pdf/ dossier_sns_2017_vdef.pdf

Déclaration de liens d'intérêts Les auteurs déclarent ne pas avoir de liens d'intérêts. 